\title{
Prospective associations between combined physical activity and sedentary behaviours and milk and yogurt consumption. Results from the IDEFICS study
}

\author{
Alba M Santaliestra Pasias ${ }^{1,2}$, Esther M. González-Gil ${ }^{1,2}$, Valeria Pala ${ }^{3}$, Timm Intemann ${ }^{4,5}$, \\ Antje Hebestreit $^{6}$, Paola Russo ${ }^{7}$, Carola Van Aart ${ }^{8}$, Patrizia Rise ${ }^{9}$, Toomas Veidebaum ${ }^{10}$, \\ Denes Molnar ${ }^{11}$, Michael Tornaritis ${ }^{12}$, Gabriele Eiben ${ }^{13,14}$ and Luis Moreno ${ }^{1,2}$ \\ ${ }^{1}$ GENUD (Growth, Exercise, NUtrition and Development) Research Group, Universidad de Zaragoza, Zaragoza, \\ Spain, \\ ${ }^{2}$ Instituto Agroalimentario de Aragón (IA2). Instituto de Investigación Sanitaria Aragón (IIS Aragón). Centro de \\ Investigación Biomédica en Red de Fisiopatología de la Obesidad y Nutrición (CIBERObn), Zaragoza, Spain, \\ ${ }^{3}$ Department of Research. Epidemiology and Prevention Unit. Fondazione IRCCS Istituto Nazionale dei Tumori, \\ Milan, Italy, \\ ${ }^{4}$ Leibniz Institute for Prevention Research and Epidemiology-BIPS, Bremen, Germany, \\ ${ }^{5}$ Institute of Statistics, University of Bremen, Bremen, Germany, \\ ${ }^{6}$ Leibniz Institute for Prevention Research and Epidemiology - BIPS, Bremen, Germany, \\ ${ }^{7}$ Institute of Food Sciences, National Research Council, Avelino, Italy, \\ ${ }^{8}$ Department of Public Health, Ghent University, Ghent, Belgium, \\ ${ }^{9}$ Department of Pharmacological Sciences. University of Milan, Milan, Italy, \\ ${ }^{10}$ National Institute for Health Development. Estonian Centre of Behavioral and Health Sciences, Tallinn, Estonia, \\ ${ }^{11}$ Department of Paediatrics. University of Pécs, Pécs, Hungary, \\ ${ }^{12}$ Research and Education Institute of Child health, Strovolos, Cyprus, \\ ${ }^{13}$ Section for Epidemiology and Social Medicine (EPSO), University of Gothenburg, Gothenburg, Sweden and \\ ${ }^{14}$ Department of Biomedicine and Public Health, University of Skövde, Skövde, Sweden
}

\section{Abstract}

Background: The knowledge about the association of physical activity (PA) and sedentary behaviours (SB) with the most considered healthy dairy products consumption, as milk and yogurt, in childhood is scared.

Aim: To assess the longitudinal relationship between specific lifestyle behaviours (PA and SB) and combined dairy consumption (milk + yogurt) in a sample of European children.

Methods: Two measurements, with 2 years' interval (T0 and T1), were conducted in 1688 (50.8\% boys) childrenfrom the IDEFICS study. Dietary information was parental-registered by a 24-hour dietary recall. At both time points, sedentary behaviour and objective estimation of PA was obtained by accelerometers. Different groups were defined according to the international children's PA and SB behaviours recommendations over time. The cut-offs for the SB and PA recommendations were established on 2 hour/day of SB and 2 hour/day of moderate to vigorous PA. Multilevel ordinal logistic regression models were used to assess the group's association with the combined dairy consumption (milk + yogurt), adjusted for potential confounders (sex, body mass index zscore, intervention versus control region, parental education level, dietary quality index, energy intake and the consumption by each dairy group at T0).

Results: Nine groups of meeting or not both recommendations (SB and PA) were obtained. Those children who meeting both lifestyle recommendations at both measurement points, had higher probability to consume more milk and yogurt ( $\mathrm{p}<0.05)$, in comparison to the rest of combinations. Those children who did not meet any recommendations at both time points were less likely to consume milk + yogurt (OR: 0.47, 95\%CI:0.26-0.83) than those who met both recommendations at both time points. Those children which improved one behaviours (PA or SB) between both measurement point were associated with low consumption of milk + yogurt (OR:0.41, 95\%CI 0.22;0.74; OR:0.40, 95\% CI 0.22,0.72), compared with those who meet both lifestyle recommendations at both periods (T0 and T1). Also, those which improved both behaviours (PA and SB) were less likely to consume milk + yogurt (OR:0.34, 95\% CI $0.15-0.80$ ) than those who were physically active and low sedentary at both time points.

Conclusions: These results suggest that European children with a healthy lifestyle regarding PA and SB over time, had the highest milk and yogurt consumption in comparison with other children.

\section{Conflict of Interest}

None 